\title{
Review
}

\section{Fundamental Considerations for Genetically- Guided Pain Management with Opioids Based on CYP2D6 and OPRM1 Polymorphisms}

Gualberto Ruaño, $\mathrm{MD}, \mathrm{PhD}^{2}$, and Jonathan A. Kost, $\mathrm{MD}^{1}$

From: ${ }^{1}$ Genomas Inc., Hartford, CT; ${ }^{2}$ Pain

Treatment Center, Hartford Hospital, West Hartford, CT

Address

Correspondence: Gualberto Ruano, MD,

$\mathrm{PhD}$

Genomas Inc. 67 Jefferson St

Hartford, CT 06106

E-mail: gualberto. ruano@hhchealth.org

Disclaimer: There was no external funding in the preparation of this manuscript. Conflict of interest: See pg. E619

Manuscript received: 01-28-2018

Revised manuscript received: 05-05-2018 Accepted for publication: 05-22-2018

Free full manuscript: wwW. painphysicianjournal. com
Background: A major challenge for effective pharmacotherapy in pain management is to provide the drug best suited to the patient's innate characteristics.

Objective: The article illustrates pharmacogenetic principles to optimize treatments for patients and increase the likelihood of pain relief without dependence. Genetic variances are particularly relevant to opioid drugs used in pain control, and can now be harvested for predictive clinical decision support.

Study Design: Clinically actionable polymorphisms in CYP2D6 (cytochrome p450 2D6) and OPRM1 ( $\mu 1$ opioid receptor), the most important gene coding, respectively, for a metabolizing enzyme and receptor for opioids are reviewed, and functional effects described.

Methods: Risk of dysfunction is calculated from the frequency of the alleles with null function for CYP2D6, and from the low function polymorphism for OPRM1. Integration of genetic variability was performed for 9 combinatorial scenarios for CYP2D6 and OPRM1. Each combination was quantified in frequency and classified for clinical impact. A rational and pharmacological basis for personalized pain management based on pharmacokinetic and pharmacodynamic modeling is extracted from the frequency of the combinations.

Results: Patients can be classified in 3 broad risk categories for opioid side effects and dependence. Patients at high-risk with dysfunctional CYP2D6 or OPRM1 account for $\sim 14 \%$ of the population and are best managed with non-opioids. Patients at medium risk with subnormal CYP2D6 or OPRM1 account for $\sim 48 \%$ of the population and can be managed with dose monitoring. Patients at low risk with functional CYP2D6 and OPRM1 account for $~ 38 \%$ of the population and should be availed to opioid therapy.

Limitations: Heuristic clinical decision support considerations are not validated yet by deployment in large clinical practices. Environmental modifiers such as other drugs and dietary supplements interact with innate characteristics to modify the genetic predictions.

Conclusion: Through clinical decision support interpreting the genotyping data, drug choices and doses can then be tailored to provide safe and effective therapy for individual patients. This precision affords personalized medicine to be practiced in pain treatment. Genetic factors could help determine why some patients seem more vulnerable than others to opioid side effects and dependence.

Key words: Pain management, opioids, CYP2D6, OPRM1, clinical decision support, pharmacokinetics, pharmacodynamics, pharmacogenetics, combinatorial genotypes

Pain Physician 2018: 21:E611-E621
$\Delta$ majorchallengeforeffectivepharmacotherapy in pain management is to provide the drug best suited to the patient's innate characteristics. Pain physicians need more information on current practices to optimize treatments for patients and increase the likelihood of pain relief without dependence. This review will bring a fundamental pharmacogenetic perspective to practical decisions on 
opioid prescription. It is being written by 2 physicians, 1 experienced in pain management for 20 years, and the other an expert in clinical pharmacogenetics, with the intention of illustrating the fundamental considerations that should be implemented now for geneticallyguided opioid management in contemporary practice.

There are many scenarios in which opioids may still be a beneficial treatment for pain, such as for postsurgical or debilitating chronic pain according to recent guidelines (1-3). In light of the recent opioid crisis, effectively treating this population of patients poses a quandary for the pain physician. We must ensure access to opioids for patients when it is medically warranted. But this access must be accomplished in a safe manner that prevents progression of the normal physiological dependence to pathological addictive disorders (4). Personalization could be valuable in curbing the opioid crisis, and genetics is a fundamental component of that personalization.

\section{Genetic Variation and Opioid Therapy}

Variability in pharmacokinetics and pharmacodynamics is evidenced at the molecular level by genetic polymorphism. The medical adages describing pharmacokinetics as "what the body does to the drug," and pharmacodynamics as "what the drug does to the body" remain useful concepts. Xenobiotic enzymes such as the cytochrome P450 (CYP450) family are the primary oxidative metabolizers of most drugs. Receptors to endogenous ligands such as the endorphins are the primary targets of opioids.

Genetic variability has accumulated in humans since prehistoric times in these enzymes and receptors resulting in strikingly different blood levels, effectiveness, and safety of drugs among individuals when a recommended dose is designed to treat the "average" person. These variances are particularly relevant to opioid drugs used in pain control and can now be measured by genotyping before treatment. Through clinical decision support interpreting the genotyping data, drug choices and doses can then be tailored to provide safe and effective therapy for individual patients. This precision affords personalized medicine to be practiced in pain treatment.

This article will describe CYP2D6 (cytochrome p450 2D6) and OPRM1 ( $\mu 1$ opioid receptor), the most important gene coding, respectively, for a metabolizing enzyme and receptor for opioids (5-11). Integration of their variability in each patient allows for building a rational and scientific basis for personalized pain management (Fig. 1). We will integrate the molecular information gained from genotyping into heuristic and practical clinical decision support considerations and provide examples of how genetic factors could help determine why some patients seem more vulnerable than others.

\section{Opioid Pharmacokinetics and CYP2D6}

We now have the ability to reliably assess the variation in the CYP2D6 gene (12). CYP2D6 is a hypervariable and hypermutable gene critically relevant to the pharmacogenetics of psychiatric and pain medications (13). CYP2D6 function is a major determinant of therapeutic response to opioids. CYP2D6 manifests duplications, rearrangements, deletions, and highly diverse haplotypes

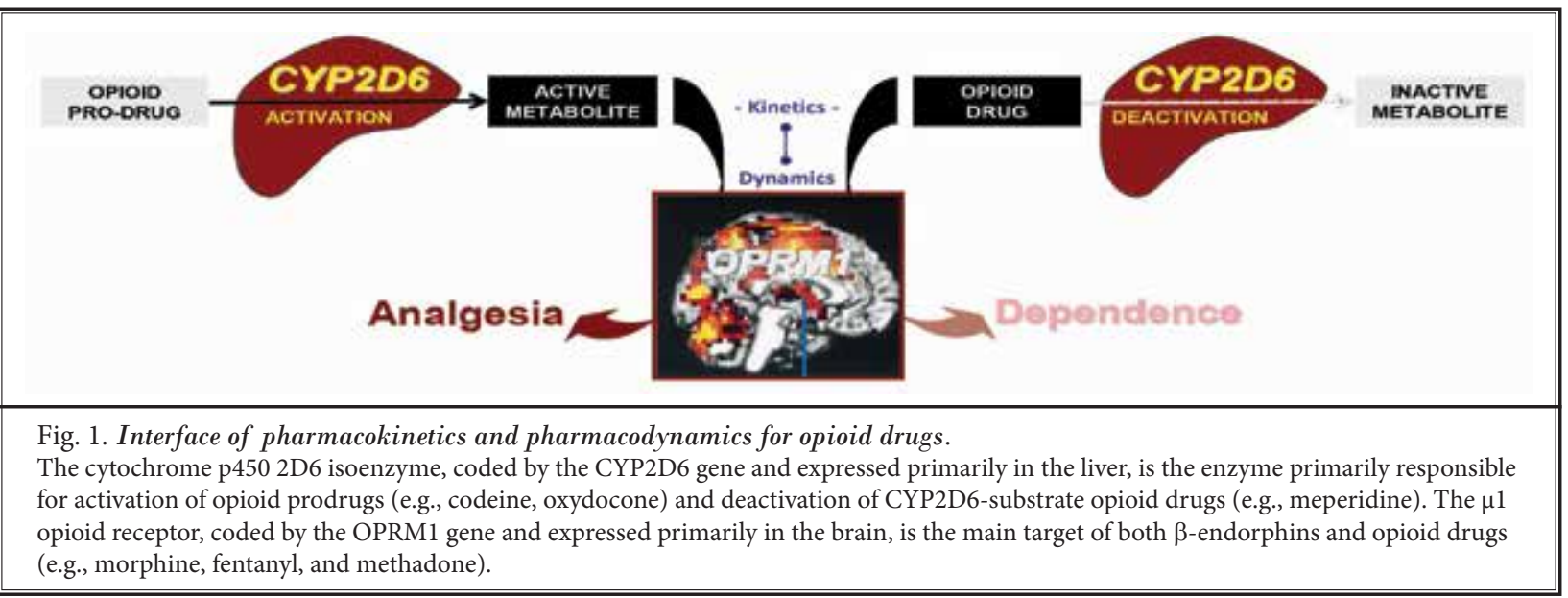


which characterize this gene (14). Besides the molecular complexity, there has been inconsistent assignment of function and nomenclature to the phenotypes (e.g. poor, intermediate, extensive, ultra-rapid) which has retarded widespread use of CYP2D6 genotyping (15). Many of the polymorphisms are bona fide mutations since these render the protein ineffectual (16). Such CYP2D 6 mutations are $* 3, * 4, * 4 X N, * 5$ (deletion), *6, $* 7, * 8, * 11, * 12, * 14, * 15$ and account for a combined frequency of $20 \%$ in a referred population of 2406 primary care and psychiatric patients (17). There are gene expansions that confer ultra-rapid CYP2D6 metabolizer status $(* 1 X N, * 2 X N)$, accounting for $3 \%$ in the same cohort resolved at high resolution by expert haplotyping procedures (17).

Both null and expansion alleles, at $23 \%$ combined total frequency, are dysfunctional, as these bring metabolizer phenotypes to the extremes of function, poor and ultra-rapid, respectively. Thus, we prefer a simpler functional logic instead that combines all the null mutations and gene expansions of CYP2D6 into a dysfunctional allele category which allows a heuristic application, and direct guidance for the clinician. There are 3 practical functional CYP2D6 phenotypes in our logic: normal, subnormal and dysfunctional. The phenotypes, allele configurations, and estimated prevalences based on the Hardy-Weinberg law are as follows:

- CYP2D6 Functional (neither CYP2D6 allele is null or ultra-rapid) at $60 \%$ of the population,

- CYP2D6 Subnormal (one CYP2D6 allele is null, the other normal) at $30 \%$,

- CYP2D6 Dysfunctional (both CYP2D6 alleles are null or at least one is ultra-rapid) at $10 \%$.

In the context of prescribing opioids for pain management, determination of CYP2D6 gene variation is key to understanding inherent suitability of the patient, because of the effects of variants on drug metabolism. It has been shown that opioids have adverse events in patients at both extremes of function, ultra-rapid $(18,19)$ and poor $(20)$. Further, in a survey of CYP2D6 metabolizer status at a specialized pain treatment center, it was found that these extremes tended to be enriched in the referred populations (21). It is for this reason, that we consider both metabolizer extremes (ultra-rapid and poor) as dysfunctional and recommend that CYP2D6 substrate drugs and prodrugs be avoided in these patients.

Codeine, oxycodone, hydrocodone, and tramadol are opioid prodrugs with limited analgesic effect on their own as ingested. These prodrugs require hepatic CYP2D6 conversion to their most active metabolite to exert analgesia (Fig. 2). A CYP2D6 poor metabolizer obtains hardly any pain relief from the opioid prodrugs. For meperidine, which is an opioid drug primarily metabolized by CYP2D6 to an inactive metabolite, an ultra-rapid metabolizer would obtain less pain relief than a patient with normal function. Morphine and methadone are minor substrates for CYP2D6 also deactivated by CYP2D6 but partially, and likely less effective in ultra-rapid metabolizers. Whether an opioid is a CYP2D6 prodrug or drug, the results in dysfunctional analgesic effects on patients is at the extremes of CYP2D6 functional status.

The ultra-rapid status presents unique risks for opioid treatments. For ultra-rapid metabolizers, prodrugs are metabolized in a burst as a bolus of active metabolite, producing serious side effects such as respiratory depression, and at best an unsustainable response. The ultra-rapid status results in immediate breakdown, which prevents the attainment of therapeutic, steadystate drug concentrations.

Published cases of opioid toxicity due to codeine prescription in CYP2D6 ultra-rapid metabolizers are illustrative of this risk. In one non-lethal case, a cancer patient with pneumonia given codeine for cough suppression went into respiratory arrest. Genotyping characterized the patient as a CYP2D6 ultra-rapid metabolizer with a functional gene expansion. Death was averted when the patient was treated with naloxone and fully recovered (22). In a tragic case, the newborn of a mother taking codeine died 13 days after birth. It was determined that breast milk from the mother, who was a CYP2D6 ultra-rapid metabolizer (with a functional gene duplication), was the inadvertent source of lethal doses of morphine (codeine's metabolite) to the baby (23). It has been suggested that codeine should be avoided by breast-feeding mothers who are ultra-rapid metabolizers of CYP2D6. The safety profile of codeine was reevaluated, and the FDA issued a Black-box warning on codeine use in nursing mothers.

For the drugs metabolized to an inactive moiety, a rapid metabolizer obtains much less pain relief than a normal metabolizer. Rapid metabolizer patients may also evidence unusual psychiatric symptoms of opioid intoxication after codeine, hydrocodone, or oxycodone such as nervousness, restlessness, confusion, hallucinations, or paradoxical stimulation. In cases of dysfunctional CYP2D6, opioids or alternative analgesics which 


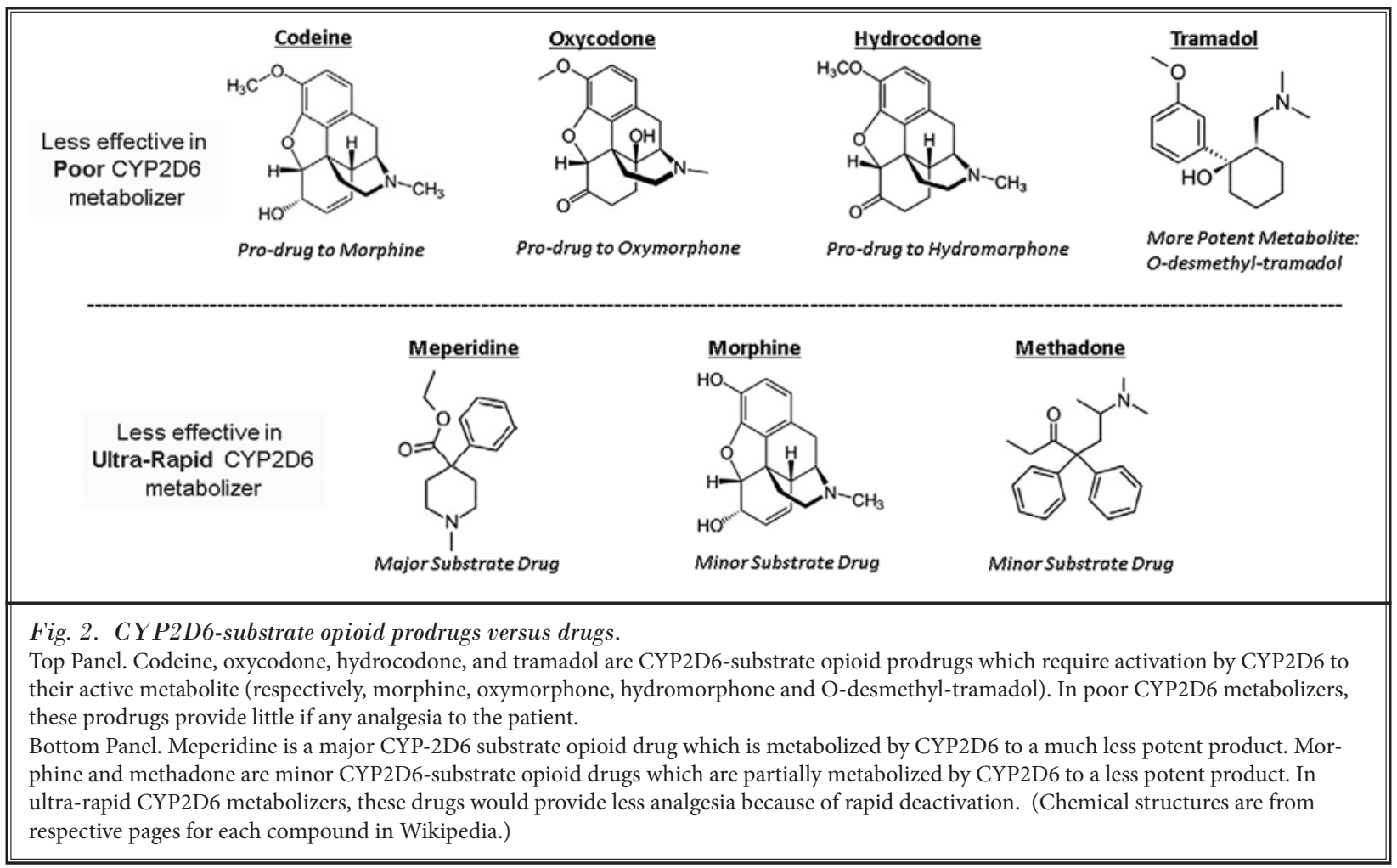

are not primary substrates of CYP2D6 should be considered. Opioids which are not CYP2D6 substrates include oxymorphone, hydromorphone, and buprenorphine (Fig. 3).

\section{Opioid Pharmacodynamics and OPRM1}

It is becoming evident that pharmacodynamic genetic variants contribute to the development of opioid dependence. The $\mu$ opioid receptor is the main target of both endogenous and clinically used opioids, such as oxycodone, their antagonists such as naltrexone, and an important mediator of drug dependence and opioid-induced respiratory depression (24). The $\mu 1$ opioid receptor gene is the most widely studied gene in association with different aspects of chronic pain.

The $\mu 1$ opioid receptor gene (OPRM1) that codes for this receptor has a functionally significant and common variant termed A118G (rs1799971) (25). This single nucleotide polymorphism (SNP) in exon 1 of the gene causes transition of an adenine (A) nucleotide to guanine (G) at base 118. In turn, the A118G transition at the DNA sequence causes the amino acid exchange at residue 40 of the $\mu$ opioid receptor protein from the normal asparagine (Asn) to an abnormal aspartic acid (Asp) residue (Asn40Asp).
The Asp40 isoform of the receptor does not carry a $\mathrm{N}$-glycosylation site in the extracellular region of the receptor, which reduces expression of the isoform at the cell surface, decreases $\mu$-receptor binding potential in the brain, and increases morphine requirement (26). Significant reduction in effectivity of subsequent signaling pathways after the binding of specific agonists has been observed. The rate of G-protein coupling in carriers of the $G$ allele is only half of that of $A A$ homozygotes.

The global average frequency of the abnormal $\mathrm{G}$ allele is $\sim 20 \%$, but with a wide population-specific range from $3 \%$ in individuals of African descent to nearly $50 \%$ in those of Asian descent. The phenotypes, allele configurations, and estimated prevalences based on the Hardy-Weinberg law are as follows for a $\mathrm{G}$ allele frequency of $20 \%$ :

- OPRM1 Functional (AA homozygous, both OPRM1 alleles are normal) at $64 \%$ of the population,

- OPRM1 Subnormal (AG heterozygous, one OPRM1 allele is normal, one abnormal) at $32 \%$,

- OPRM1 Dysfunctional (GG homozygous, both OPRM 1 alleles are abnormal) at $4 \%$. 


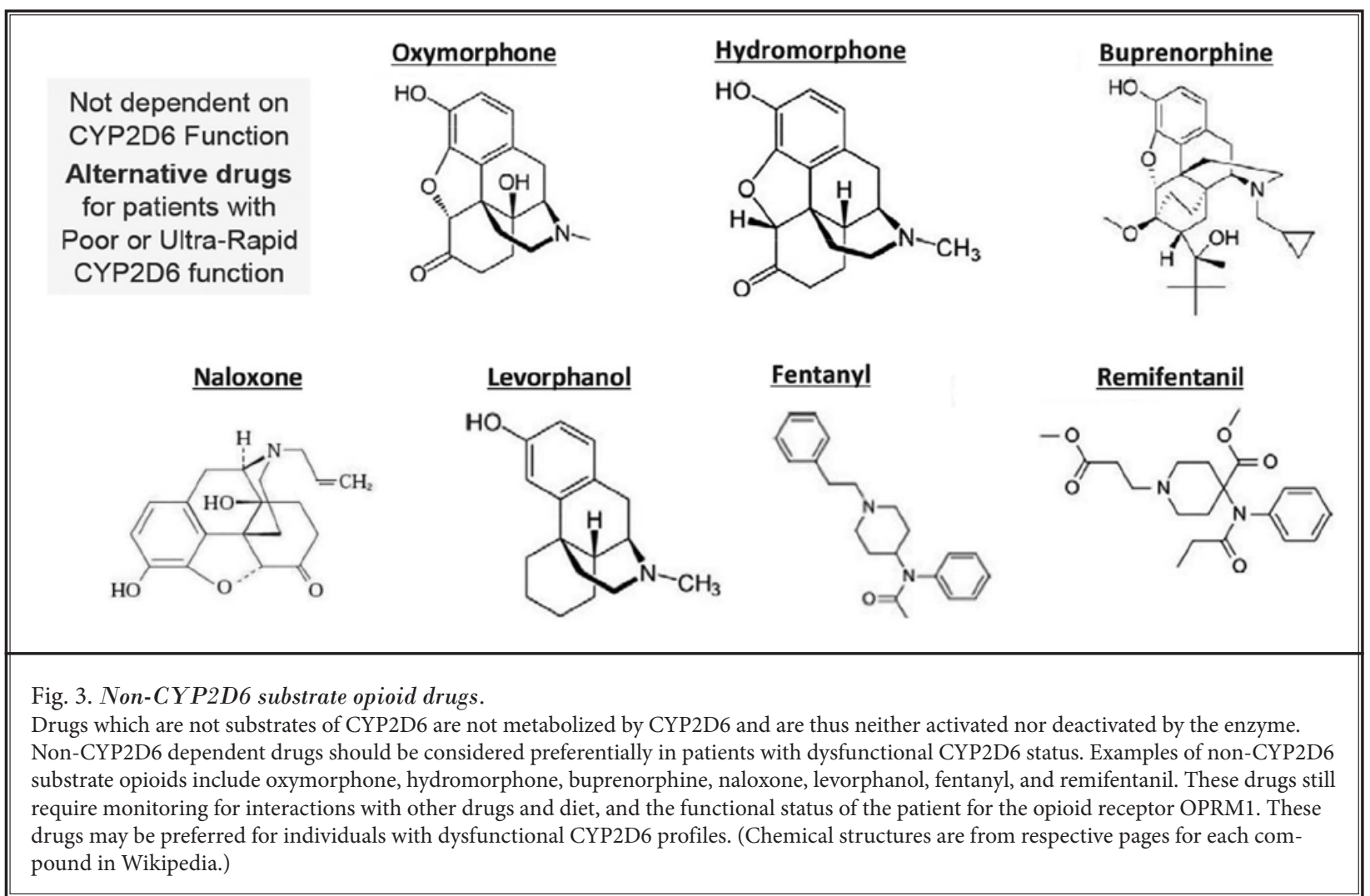

The respective isoform configurations at Asn40Asp are Asn/Asn (homozygous, functional), Asn/Asp (heterozygous, subnormal) and Asp/Asp (homozygous, dysfunctional).

Several studies show evidence that having at least 1 copy of the G allele (AG or GG) is associated with lower pain threshold, and higher opioid consumption in post-operative patients. Thus, this common protein coding polymorphism found in OPRM1 could render a patient less sensitive to opioid analgesic effects, and more prone to dependence (27). In various clinical scenarios, patients with the $G$ risk allele (Asp), rather than the normal A allele (Asn), appeared less sensitive to opioid medications. It is a trait that negatively could affect patient outcomes to opioid treatments.

\section{Clinical Considerations and Decision SUPPORT}

We prefer heuristic grounds and "rules of thumb" for integrating these results based on basic principles of pharmacokinetics and pharmacodynamics. The rationale is based on observing the combinatorial configura- tions of CYP2D6 and OPRM1 in a given patient (Table 1). Considering the 3 functional states for each CYP2D6 and OPRM1 gene, there are 9 combinatorial categories.

- If a patient has functional CYP2D6 and OPRM1 phenotypes, the physician has carte blanche to implement judgment and experience garnered from previous patients.

- When CYP2D6 is subnormal in a patient, we suggest implementing dosing restrictions determined by whether the medication is a drug or prodrug. If CYP2D6 is dysfunctional, we recommend nonCYP2D6 substrate drugs.

- When a patient has OPRM1 subnormal or dysfunctional, the patient is less likely to benefit from opioids. There would be a higher incidence of side effects and dependence risk as well for these patients. We carefully monitor opioid doses in OPRM1 subnormal patients.

One would avoid escalating opioid doses if a patient reports inadequate pain relief. We recommend avoid- 
Table 1. Set of 9 combinatorial functional configurations of CYP2D6 and OPRM1 based on a tri-fold functional status for each.

\begin{tabular}{|c|c|c|c|c|c|c|}
\hline$\#$ & CYP2D6 & $\begin{array}{l}\text { CYP2D6 } \\
\text { Prodrugs }\end{array}$ & $\begin{array}{l}\text { CYP2D6 } \\
\text { Drugs }\end{array}$ & OPRM1 & $\begin{array}{c}\text { OPRM1 } \\
\text { Opioid Risk? }\end{array}$ & Freq. \\
\hline 1. & Functional & Normal dose & Normal dose & Functional & Low & $38.4 \%$ \\
\hline 2. & Subnormal & High dose & Low dose & Functional & Low & $19.2 \%$ \\
\hline 3. & Dysfunctional & Non-CYP2D6 & Non-CYP2D6 & Functional & Low & $6.4 \%$ \\
\hline 4. & Functional & Normal dose & Normal dose & Subnormal & Moderate & $19.2 \%$ \\
\hline 5. & Subnormal & High dose & Low dose & Subnormal & Moderate & $9.6 \%$ \\
\hline 6. & Dysfunctional & Non-CYP2D6 & Non-CYP2D6 & Subnormal & Moderate & $3.2 \%$ \\
\hline 7. & Functional & Normal dose & Normal dose & Dysfunctional & High & $2.4 \%$ \\
\hline 8. & Subnormal & High dose & Low dose & Dysfunctional & High & $1.2 \%$ \\
\hline 9. & Dysfunctional & Non-CYP2D6 & Non-CYP2D6 & Dysfunctional & High & $0.4 \%$ \\
\hline
\end{tabular}

Green indicates a Functional status for the gene (CYP2D6 -- neither allele is null or ultra-rapid; OPRM1 -- both alleles are normal, AA). Yellow indicates Subnormal status (CYP2D6 -- one allele is null, the other normal; OPRM1 -- one allele is abnormal, the other normal, AG). Red indicates Dysfunctional status (CYP2D6 -- both alleles are null or at least one is ultra-rapid; OPRM1 -- both alleles are abnormal, GG).

Guidance is provided for prescription, dosing, and selection of opioids, based on the combinatorial functional configuration. The estimated prevalence of each combinatorial configuration is based on the multiplication of individual frequencies for the functional categories of both genes (CYP2D6 X OPRM1).

The CYP2D6 enzyme is the primary activator of codeine, oxycodone, hydrocodone, and tramadol, which are termed prodrugs because their metabolites are more potent analgesics than the parent molecule. CYP2D6 is the primary inactivator of meperidine and a minor one of morphine and methadone, which are therefore conventional drugs, more potent than any of their respective metabolites.

ing opioids altogether in OPRM1 dysfunctional patients and rely on non-opioids. Alternative electrophysiological and psychological therapies could be most useful in patients for whom drug therapy with opioids is ineffective or unsafe. Fortunately, modern pain management does also afford non-pharmacological treatments.

We can summarize these approaches into 3 clinical categories and priorities for genetically-guided opioid management.

- Clinical Category and Priority \#1. Patients at high-risk with dysfunctional CYP2D6 or OPRM1 account for $\sim 14 \%$ of the population [categories $3(6.4 \%), 6(3.2 \%), 7(2.4 \%), 8(1.2 \%), 9(0.4 \%)$ in Table 1]. These patients must be identified early, and may be treated with alternatives to opioids.

- Clinical Category and Priority \#2. Patients requiring opioid dose adjustment with subnormal CYP2D6 or OPRM1 account for $\sim 48 \%$ of the population [categories $2(19.2 \%), 4(19.2 \%), 5$ (9.6\%) in Table 1]. These patients are important to recognize for adjustments in opioid selection, dosing, and monitoring of response.

- Clinical Category and Priority \#3. Patients likely to respond to standard opioid prescription and dosing with functional CYP2D6 and OPRM1 account for $38 \%$ of the population [category 1 (38.4\%) in Table 1]. These are the patients who would suffer the most if disqualified from opioid therapy based on uninformed draconian prescription restrictions. 


\section{Gene-Environment Interactions}

Personalizing pain management requires screening not only for opioid genetic alterations in the patient but also for interfacing environmental triggers that interact with the gene targets. Drug and diet interactions with metabolism inhibitors and inducers are the preeminent environmental modifiers in the pharmacokinetic dimension. Co-prescription of any CYP2D6 strong inhibitors could generate an equivalent subnormal or dysfunctional phenotype and should be safeguarded with caution when opioids are being prescribed (28-30). Strong CYP2D6 inhibitors include antidepressants (e.g., bupropion, fluoxetine, paroxetine), antifungals (e.g., ketoconazole, miconazole) and antivirals (e.g., delavirdine, ritonavir). Dietary interactions are also significant, particularly with herbals (31-33). Some common dietary supplements interact with CYP450 and inhibit CYP2D6 (e.g., sesamin, turmeric). Lotus herbals (e.g., cosmetics, teas) significantly inhibit CYP2D6.

There are important clinical correlations that should provide the pharmacodynamic dimension for assessing opioid tolerance and dependence. Validated patient outcomes tools for pain management include the PEG-3 (Pain, Enjoyment, General Activity) Scale, COMM-9 (Current Opioid Misuse Measure), and SOAPP (Screener and Opioid Assessment for Patients with Pain) (34-37). As with all clinical symptoms, these represent the environmental modifiers. The genetic analysis offers perspective on the innate constitution of the individual as a baseline on which to superimpose environmental effects.

Toxicology urine drug screens are routine and valuable for monitoring the composite of environmental and clinical determinants of a patient's treatment. However, these are not sufficient for monitoring opioids to determine the patient's compliance or dependence. Even when performed at high resolution with mass spectrometry, urine toxicology will not be useful for phenotype-to-genotype correlations until the toxicology and genetic data are integrated into a clinical decision support system. Because of individual CYP2D6 metabolizer status, a fully compliant patient who is an ultra-rapid metabolizer could test negative for an opioid while a poor metabolizer could test consistently above the normal range.

\section{Limitations in SCOPE fOR FUtURE Consideration}

In integrating pharmacokinetics and pharmacodynamics, this article has considered some very complex pathways of drug metabolism and activation while obviating others. The role of CYP3A4 in opioid metabolism is very significant for fentanyl, which is a major substrate, and secondary to CYP2D6 for codeine, hydrocodone, oxycodone and tramadol (38). CYP3A4 enzyme can be inhibited and the gene induced by multiple other drugs and diet. For example, macrolide antibiotics, azole antifungals, protease inhibitors and citrus juices are strong inhibitors, while rifamycins and anticonvulsants are strong inducers (5). CYP3A4 is far less genetically variable than CYP2D6, and is best managed by monitoring inhibitors and inducers that may be co-prescribed. This dimension is outside the scope of this article focused on genetic variation, but will be broached in a future review.

This article addressed only Phase I pharmacokinetics, and did not included consideration of Phase II and glucuronidation. In this domain, uridine diphosphoglucuronosyltransferase 2B7 (UGT2B7) is the predominant enzyme responsible for the glucuronidation of morphine to morphine-6-glucuronide (M6G) and morphine-3-glucuronide (M3G). The analgesic properties of morphine are enhanced by M6G and reduced by M3G. Polymorphisms in the gene encoding UGT2B7 may, therefore, have pharmacological, toxicological, and physiological significance (39).

As an example of the critical role of CYP2D6 genetic variation over CYP3A4 and UGT2B7, is the fact that the Clinical Pharmacogenetics Implementation Consortium guidance on opioids and CYP2D6 has a clear delineation of ultra-rapid and poor CYP2D6 function as a major risk for codeine prescription (40). The guidance advises avoiding codeine altogether for patients with these extreme phenotypes, and suggests that to avoid treatment complications "opioids that are not metabolized by CYP2D6, including morphine, oxymorphone, buprenorphine, fentanyl, methadone, and hydromorphone, along with nonopioid analgesics, may be considered as alternatives for use in CYP2D6 poor and ultrarapid metabolizers". It further cautions that "tramadol and, to a lesser extent, hydrocodone and oxycodone are not good alternatives because their metabolism is affected by CYP2D6 activity."

The classical distinction between pro-drugs and drugs rarely applies absolutely to opioids because both the precursor molecule and the metabolite are pharmacologically active to some degree. Nevertheless, CYP2D6 is the primary activator of codeine, oxycodone, hydrocodone, and tramadol whose metabolites are more potent analgesics than the parent molecule. Con- 
versely, CYP3A4 is not involved in any conversion of a parent opioid to a more potent metabolite. Hence, for this initial exploration of genetic guidance for opioid prescription, CYP2D6 is the gene whose polymorphisms are of greatest clinical importance.

\section{Further Research and Multi-gene Panels}

There is promising research indicating that other CYP450 genes (e.g., CYP3A4), opioid receptors (e.g., $\delta$ - and $\kappa$ - receptors; genes OPRD1, OPRK1) and dopaminergic targets (e.g., dopamine receptor D2, transporter, and $\beta$-hydroxylase; genes DRD2, SLC6A3, DBH) may also contribute to a multi-gene model of response (41-43). However, functional variability in CYYP3A4 is primarily dependent on inhibition or induction by other drugs, rather than genetic polymorphism (5). Further, it has been demonstrated that among the opioid receptors, OPRM1 is the predominant predictor of opioid dependence. OPRM1 was involved in 17 of 18 models derived to predict opioid dependence (and in all the 10 most statistically significant ones), while OPRD1 was involved in 13, and OPRK1 in 6 (44).

Including more genes in a model does not necessarily mean better prediction (45). As the roster of genes is increased, so do the possible environmental modifiers and disease comorbidities which may mask the innate gene effect of less predictive genes (46-47). Hence, multi-gene models invariably require very large populations to determine and validate the appropriate coefficients for each gene in a predictive matrix.

Some of these gene panels and interpretative algorithms are available in the market from commercial vendors (48). While genetic approaches may be represented in the commercial marketplace as a panacea, we believe physicians should also be informed about the inherent predictive limitations in models from vendors of multi-gene panels. Unfortunately many of the algorithms in the market are maintained as trade secrets by the vendors, rendering it impossible for the physician to assess the predictive power or validity of the components in what effectively is a "black box." Such overmarketing eventually backfires because uninformed utilization, and increased costs lead to restrictions in coverage by insurance companies, and to limitations in access for clinicians and patients.

Thus, even when such a multi-gene model has been obtained for a patient, we recommend that the physician consider each gene result individually. The configuration of the main opioid pharmacokinetic and pharmacodynamic genes, CYP2D6 and OPRM1, should be assessed carefully because these remain the genes with the highest effect size and clinically predictive value based on the functional status as derived from their respective genotypes.

\section{Personalization of Regimens to Combat the Opioid Crisis}

Personalization is important for all patients, but essential for those requiring preparation for starting or reversing a regimen of high-dose opioids or an unusual combination of agents. According to the National Center for Health Statistics, more than 63,600 lives were lost to drug overdose in 2016, the most lethal year yet of the drug overdose epidemic. Most of those deaths, 42,249 fatalities or $66 \%$, involved opioids (including heroin overdoses). Opioid abuse is the leading cause of mortality for people under age 50 (49).

The current opioid crisis represents an alarming shift in the real outcomes of pain management from the patient's improvement in quality of life to iatrogenic drug dependence and addiction (50-52). Integral to the efficient and effective remediation of this crisis is the practice of personalized pain management, defined as the integrative assessment of clinical and genetic data for each individual. Legislative or legal moratoria on opioid use without prescription personalization can harm the public health at large by preventing physicians from treating the patients who can be genetically predicted to benefit the most from an important medication class.

Cost-benefit considerations of pharmacogenetic testing should recognize the current reality that many primary care physicians are resistant to treating pain patients and are opting to refer the patients to pain management specialty clinics. The evidence for the cost-effectiveness of pharmacogenetic testing relies on modeling potential savings versus the cost of genotyping. Currently, the current cost of genotyping hovers at $\sim 500$ per patient, which is high (21). However, genotyping can be effective when its cost is counterbalanced by savings in the health care system and services. Studies examining clinic and prescription usage are under consideration. Drug waste from prescription changes, and therapeutic management complications stemming from dysfunctional status could be avoided by pharmacogenetic guidance. The savings realized from genotyping could amortize the cost of testing, which itself is likely to come down. It is possible to devise clinical criteria to select patients for genotyping and limit the testing initially to those with opioid intolerance, 
side effects or ineffectiveness to reduce the total cost of genotyping. The current claim-based reimbursement restrictions on a per test basis remain a hurdle to the widespread implementation of gene-guided pain management. We foresee that evolving models for valuebased reimbursement may remedy this problem, as genotyping becomes incorporated into the total package of care, including costs, revenue and profit.

Data storage, health informatics and reporting system that enable physicians to access patient outcomes and test results immediately following completion of treatments and laboratory processing are necessary for the personalization of pain management. Such immediate and interactive presentation of outcomes and test results in an unambiguous, accessible and clinically actionable format will facilitate the translation of clinical data and laboratory results (including toxicology and genetics) to personalized pain management.

We have discussed practical guidelines for implementation of CYP2D6 and OPRM1 polymorphisms for genetically-guided opioid prescription. Within the realm of utility, genetic predictions of opioid pharmacokinetcs and pharmacodynamics are among the most accurate and clinically actionable because these reflect drug-gene interactions. These offer risk-management stratification to safeguard the patients who are least likely to benefit from opioids or who require a greater degree of oversight and monitoring while on opioids while identifying the others who are likely to respond and may be deprived of the most potential benefit if opioids are unduly restricted.

\section{Conclusion}

In conclusion, we posit that the personalization of pain management offers a new promising dimension that has been overlooked so far when confrontingthe opioid crisis, and that genetics is a key component of that personalization. Genetics offers objective information on the innate baseline of the individual, upon which clinical characteristics and environmental modifiers could be integrated. The potential impact is a substantial reduction of dependence, and an increased efficacy of pharmacotherapy in the field of pain management.

\section{Acknowledgments}

\section{Funding}

This work is part of general educational and scholarship activity for the authors.

\section{Conflicts of Interest}

JAK is Chief Executive Officer and Founder of ScopeData LLC. GR is Medical Director and President of Genomas Inc.

\section{Author Contributions}

GR performed the review of the pharmacokinetics and pharmacodynamics of opioids, and compiled the pharmacogenetic data for CYP2D6 and OPRM1. He calculated the combinatorial frequencies of the various alleles and classified their pharmacological significance. JAK provided the clinical annotations with regard to the opioid crisis, decision support requirements in pain management at a large practice and environmental modifiers of opioid response from drug/diet interactions.

\section{REFERENCES}

1. Nuckols TK, Anderson L, Popescu I, Diamant AL, Doyle B, Di Capua P, Chou R. Opioid prescribing: A systematic review and critical appraisal of guidelines for chronic pain. Ann Intern Med 2014; 160:38-47.

2. Dowell D, Haegerich TM, Chou R. CDC guideline for prescribing opioids for chronic pain-United States. JAMA 2016; 315:1624-1645.
3. McLellan AT. New Department of Veterans Affairs and Department of Defense Guidelines on Pain Management with Opioids: Comment and Concern. Ann Intern Med 2017; 167:50-51.

4. Volkow ND, McLellan AT. Opioid abuse in chronic pain-misconceptions and mitigation strategies. NEJM 2016; 374:1253-1263.
5. Wilkinson GR. Drug metabolism and variability among patients in drug response. NEJM 2005; 352:2211-2221.

6. Somogyi AA, Barratt DT, Coller JK. Pharmacogenetics of opioids. Clinical Pharmacology Therapeutics 2007; 81 :429-444.

7. Reynolds KK, Ramey-Hartung B, Jortani $\mathrm{SA}$. The value of $\mathrm{CYP}_{2} \mathrm{D} 6$ and $\mathrm{OPRM}_{1}$ pharmacogenetic testing for opioid therapy. Clinics in Laboratory Medicine 
2008; 28:581-598.

8. Světlík $\mathrm{S}$, Hronová $\mathrm{K}$, Bakhouche $\mathrm{H}$, Matoušková O, Slanař O. Pharmacogenetics of chronic pain and its treatment. Mediators of inflammation 2013; 864319:1-23.

9. Kapur BM, Lala PK, Shaw JL. Pharmacogenetics of chronic pain management. Clinical Biochemistry 2014; 47:1169-1187.

10. Manworren RC, Ruaño G, McGrath JM. Translating the human genome to manage pediatric postoperative pain. J Pediatr Surg Nurs 2015; 4:28-39.

11. Pergolizzi JV, LeQuang JA, Berger GK, Raffa RB. The basic pharmacology of opioids informs the opioid discourse about misuse and abuse: A review. Pain and Therapy 2017; 6:1-16.

12. Gaedigk A, Simon SD, Pearce RE, Bradford LD, Kennedy MJ, Leeder JS. The CYP2D6 activity score: Translating genotype information into a qualitative measure of phenotype. Clinical Pharmacology Therapeutics 2008; 83:234-242.

13. Skierka JM, Walker DL, Peterson SE, O'Kane DJ, Black JL. CYP2D6* 11 and challenges in clinical genotyping of the highly polymorphic CYP2D6 gene. Pharmacogenomics 2012; 13:951-954.

14. Black JL, Walker DL, O'Kane DJ, Harmandayan M. Frequency of undetected CYP2D6 hybrid genes in clinical samples: Impact on phenotype prediction. Drug Metabolism and Disposition 2012; 40:111-119.

15. Gaedigk A. Complexities of CYP2D6 gene analysis and interpretation. Int Rev Psychiatry 2013; 25:534-553.

16 Kevin-Hicks J, Swen JJ, Gaedigk A. Challenges in $\mathrm{CYP}_{2} \mathrm{D} 6$ phenotype assignment from genotype data: A critical assessment and call for standardization. Curr Drug Metab 2014; 15: 218232.

17. Ruaño G, Kocherla M, Graydon JS, Holford TR, Makowski GS, Goethe JW. Practical interpretation of CYP2D6 haplotypes: Comparison and integration of automated and expert calling. Clinica Chimica Acta 2016; 456:7-14.

18. de Leon J, Dinsmore L, Wedlund P. Adverse drug reactions to oxycodone and hydrocodone in CYP2D6 ultrarapid metabolizers. J Clin Psychopharmacol 2003; 23:420-421.

19. Ruaño G, Larsen K, Kocherla M, Graydon JS, Kost J. Complications of psychotropic and pain medications in an ultrarapid metabolizer patient at the upper $1 \%$ of cytochrome $\mathrm{P}_{450}$ (CYP450) function quantified by combinatorial CYP450 genotyping. J Pain Palliat Care Pharmacother 2017; 31:126-138.

20. Susce MT, Murray-Carmichael E, de Leon J. Response to hydrocodone codeine and oxycodone in a CYP2D6 poor metabolizer. Progress in Neuro-Psychopharmacology and Biological Psychiatry 2006; 30:1356-1358.

21. Manworren RCB, Jeffries L, Pantaleao A, Seip R, Zempsky WT, Ruaño G. Pharmacogenetic testing for analgesic adverse effects: Pediatric case series. Clin ] Pain 2016; 32:109-115.

22. Gasche Y, Daali Y, Fathi M, Chiappe A Cottini S, Dayer P, Desmeules J. Codeine into ication associated with ultrarapid CYP2D6 metabolism. NEJM 2004; 351:2827-2831.

23. Koren G, Cairns J, Chitayat D, Gaedigk A, Leeder SJ. Pharmacogenetics of morphine poisoning in a breastfed neonate of a codeine-prescribed mother. Lancet 2006; 368:704.

24. Oroszi G, Anton RF, O'Malley S, Swift R, Pettinati H, Couper D, Goldman D. $\mathrm{OPRM}_{1}$ Asn4oAsp predicts response to naltre one treatment: A haplotype-based approach. Alcoholism: Clinical and Experimental Research 2009; 33:383-393.

25. Mura E, Govoni S, Racchi M, Carossa V, Ranzani GN, Allegri M, van Schaik RH. Consequences of the $118 \mathrm{~A}>\mathrm{G}$ polymorphism in the OPRM gene: Translation from bench to bedside? ] Pain Res 2013; 6:331-353.

26. Crist RC, Berrettini WH. Pharmacogenetics of OPRM1. Pharmacology Biochemistry and Behavior 2014; 123:25-33.

27. Bauer IE, Soares JC, Nielsen DA. The role of opioidergic genes in the treatment outcome of drug addiction pharmacotherapy: A systematic review. Am J Addict 2015; 24:15-23.

28. Gressier F, Verstuyft C, Hardy P, Becquemont L, Corruble E. Response to $\mathrm{CYP}_{2} \mathrm{D6}$ substrate antidepressants is predicted by a $\mathrm{CYP}_{2} \mathrm{D} 6$ composite phenotype based on genotype and comedications with $\mathrm{CYP}_{2} \mathrm{D} 6$ inhibitors. ] Neural Transmission 2015; 122:35-42.

29. Xue C, Zhang X, Cai W. prediction of drug-drug interactions with bupropion and its metabolites as CYP2D6 inhibitors using a physiologically-based pharmacokinetic model. Pharmaceutics 2018; 10:1-20.

30. Johnson MI, Radford H. CYP2D6 polymorphisms and response to codeine and tramadol. Analgesia \& Resuscitation:
Current Research 2016; 5:1-3

31. Ye LH, HeXX, Kong LT, Liao YH, Pan RL, Xiao BX, Liu XM, Chang Q. Identification and characterization of potent $\mathrm{CY}$ P2D6 inhibitors in lotus leaves. Journal of Ethnopharmacology 2014; 153:190-196.

32. Sasaki T, Sato Y, Kumagai T, Yoshinari K, Nagata K. Effect of health foods on cytochrome $\mathrm{P} 450$-mediated drug metabolism. Journal of Pharmaceutical Health Care and Sciences 2017; $3: 14$.

33. Brewer CT, Chen T. Hepatotoxicity of Herbal Supplements Mediated by Modulation of Cytochrome $\mathrm{P}_{450}$. International Journal of Molecular Sciences 2017; 18:2353.

34. Butler SF, Budman SH, Fanciullo GJ, Jamison RN. Cross validation of the Current Opioid Misuse Measure (COMM) to monitor chronic pain patients on opioid therapy. Clin J Pain 2010; 26:770-776.

35. Passik SD, Narayana A, Yang R. Aberrant drug-related behavior observed during a 12-week open-label e tension period of a study involving patients taking chronic opioid therapy for persistent pain and fentanyl buccal tablet or traditional short-acting opioid for breakthrough pain. Pain Medicine 2014; 15:1365-1372.

36. Bruehl S, Burns JW, Passik SD, Gupta R, Buvanendran A, Chont M, France CR. The contribution of differential opioid responsiveness to identification of opioid risk in chronic pain patients. J Pain 2015; 16:666-675.

37. Eichorn D, Lesenskyj A, Ma well C, Moore S, Cruciani R. The feasibility and value of using the SOAPP ${ }^{\circledR}-R$ to predict substance abuse in an outpatient pain clinic. J Pain 2016; 17:S24.

38. Holmquist GL. Opioid metabolism and effects of cytochrome P450. Pain Medicine 2009; 10: $\mathrm{S}_{20} \mathrm{O} \mathrm{S}_{2} \mathrm{~g}$

39. Owusu Obeng A, Hamadeh I, Smith M. Review of Opioid Pharmacogenetics and Considerations for Pain Management. Pharmacotherapy. The Journal of Human Pharmacology and Drug Therapy 2017; 37:1105-1121.

40. Crews KR, Gaedigk A, Dunnenberger HM, Leeder JS, Klein TE, Caudle KE, Haidar CE, Shen DD, Callaghan JT, Sadhasivam S, Prows CA. Clinical pharmacogenetics implementation consortium guidelines for cytochrome $\mathrm{P}_{450}$ 2D6 genotype and codeine therapy: 2014 update. Clinical Pharmacology \& Therapeutics 2014; 95:377-382.

41. Cevoli S, Mochi M, Scapoli C, Marzocchi 
N, Pierangeli G, Pini L.A, Cortelli Pand Montagna P. A genetic association study of dopamine metabolism-related genes and chronic headache with drug abuse. Eur J Neurol 2006; 13:1009-1013.

42. Zhang $H$, Kranzler HR, Yang BZ, Luo X, Gelernter J. The OPRD1 and OPRKı loci in alcohol or drug dependence: OPRD variation modulates substance dependence risk. Molecular Psychiatry 2008; 13:531-543.

43. Treister R, Pud D, Ebstein RP, Laiba E, Gershon E, Haddad M, Eisenberg E. Associations between polymorphisms in dopamine neurotransmitter pathway genes and pain response in healthy humans. Pain 2009; 147:187-193.

44. Li Z, Zhang H. Analyzing interaction of $\mu$ - $\delta$-and $\kappa$-opioid receptor gene variants on alcohol or drug dependence using a pattern discovery-based method. Journal of Addiction Research Therapy Suppl 2013; 7:7-19.
45. Ruaño G. Personalizing public health in the medical laboratory: The interplay of microarrays and physiological genomics. Medical Laboratory Observer 2013; 45:36-37.

46. Cargnin S, Viana M, Sances G, Bianchi M, Ghiotto N, Tassorelli C, Nappi G, Canonico PL, Genazzani A, Terrazzino $S$. Combined effect of common gene variants on response to drug withdrawal therapy in medication overuse headache. European Journal of Clinical Pharmacology 2014; 70:1195-1202.

47. Lalanne L, Ayranci G, Kieffer BL, Lutz PE. The kappa opioid receptor: From addiction to depression and back. Frontiers in Psychiatry 2014; 5:1-17.

48. Farah JR, Lee C, Kantorovich S, Smith GA, Meshkin B, Brenton A. Evaluation of a predictive algorithm that detects aberrant use of opioids in an addiction treatment centre. Journal of Addiction Re- search Q Therapy 2017; 8:1-6.

49. Hedegaard $H$, Warner M, Miniño AM. Drug overdose deaths in the United States, 1999-2016. NCHS Data Brief(National Center for Health Statistics, Hyattsville MD) 2017; 294:1-7.

50. Keller CE, Ashrafioun L, Neumann AM, Van Klein J, Fo CH, Blondell RD. Practices perceptions and concerns of primary care physicians about opioid dependence associated with the treatment of chronic pain. Substance Abuse 2012; 33:103-13.

51. Compton WM, Boyle M, Wargo E. Prescription opioid abuse: Problems and responses. Preventive Medicine 2015; 80:5-9

52. Lin LA, Bohnert AS, Kerns RD, Clay MA, Ganoczy D, Ilgen MA. Impact of the opioid safety initiative on opioid-related prescribing in veterans. Pain 2017; 158:833-839. 
